\title{
Transtornos mentais e comportamentais no Sistema de Informações Hospitalares do SUS (SIH-SUS) no estado do Rio de Janeiro no período de 1999 a 2010
}

\author{
Mental and behavioral disorders in Hospital Information System of the \\ Unified Health System (SIH-SUS) in the state of Rio de Janeiro in the \\ period of 1999 to 2010
}

\author{
Priscila Krauss Pereira1, Simone Agadir Santos', Lúcia Abelha Lima², Letícia Fortes Legay³, \\ Jacqueline Fernandes de Cintra Santos ${ }^{4}$, Giovanni Marcos Lovisi ${ }^{5}$
}

\begin{abstract}
Resumo
O objetivo do presente artigo foi realizar uma análise epidemiológica das internações psiquiátricas do Sistema Único de Saúde (SUS) no estado do Rio de Janeiro. Os dados das internações por transtornos mentais e comportamentais foram coletados do Sistema de Informações Hospitalares (SIH-SUS) no período de 1999 a 2010. A grande maioria dos pacientes era do sexo masculino na faixa etária de 30 a 49 anos e os diagnósticos mais frequentes foram: esquizofrenia, transtornos devido ao uso de álcool e transtornos do humor. Foi observado que o número de internações por transtornos mentais apresentou queda de $70 \%$ ao longo do período estudado. As internações por transtornos mentais corresponderam a cerca de 10\% do total de hospitalizações ocorridas no estado do Rio de Janeiro, representando o segundo maior gasto com internações e perdendo apenas para as doenças do aparelho circulatório. Dessa forma, o presente artigo reforça que a utilização dos SIS em pesquisas na área da saúde mental pode ser útil não só para prover dados secundários de transtornos mentais, como também para avaliar a efetividade das políticas públicas implementadas e planejar novas ações de saúde.
\end{abstract}

Palavras-chave: transtornos mentais; hospitalização; unidades de internação; sistemas de informação; Sistema Único de Saúde.

\begin{abstract}
The aim of this study was to carry out an epidemiological analysis of the psychiatric hospitalizations in the Unified Health System (SUS) in Rio de Janeiro State. Data of hospitalization for mental and behavioral disorders were collected from the Hospital Information System (SIH-SUS) between 1999 and 2010. The majority of the patients was male, from 30 to 49 years old and the most frequent diagnosis were schizophrenia, alcohol use and mood disorders. We observed the number of hospitalizations for mental disorders decreased $70 \%$ in the studied period. The hospitalizations for mental disorders accounted for about $10 \%$ of all hospitalizations in the state of Rio de Janeiro, representing the second highest expending, after cardiovascular diseases. Therefore, this article reinforces that the SIH-SUS may be useful in mental health research area not only for providing secondary data of mental disorders, but also to evaluate the effectiveness of public policies implemented, as well to plan new health actions. Keywords: Mental disorders; hospitalization; inpatient care units; information systems; Unified Health System.
\end{abstract}

Trabalho realizado no Instituto de Estudos em Saúde Coletiva da Universidade Federal do Rio de Janeiro (UFRJ) - Rio de Janeiro (RJ), Brasil.

'Doutoranda do Programa de Pós-graduação em Saúde Coletiva do Instituto de Estudos em Saúde Coletiva da Universidade Federal do Rio de Janeiro (UFRJ) - Rio de Janeiro (RJ), Brasil.

2Doutora em Saúde Pública pela Fundação Oswaldo Cruz; Professora Adjunta do Instituto de Estudos em Saúde Coletiva da UFRJ - Rio de Janeiro (RJ), Brasil. ${ }^{3}$ Doutora em Saúde Pública pela Universidade de São Paulo (USP). Professora Associada da UFRJ - Rio de Janeiro (RJ), Brasil.

${ }^{4}$ Doutora em Saúde Coletiva pelo Instituto Fernandes Figueira. Professora Adjunta do Instituto de Estudos em Saúde Coletiva da UFRJ - Rio de Janeiro (RJ), Brasil.

${ }^{5}$ Pós-doutorado em Saúde Pública pelo Instituto de Psiquiatria da Universidade de Londres. Professor Adjunto do Instituto de Estudos em Saúde Coletiva da UFRJ - Rio de Janeiro (RJ), Brasil.

Endereço para correspondência: Priscila Krauss Pereira - Instituto de Estudos em Saúde Coletiva da UFRJ - Praça Jorge Machado Moreira - Cidade Universitária - CEP: 21944-970 - Rio de Janeiro (RJ), Brasil - E-mail: priscilakrauss@ig.com.br

Fonte de financiamento: nenhuma.

Conflito de interesse: nada a declarar. 


\section{INTRODUÇÃO}

As internações hospitalares têm sido objeto de investigação de diversos estudos, visto que constituem uma importante fonte de dados sobre morbidades de indivíduos hospitalizados, além do fato de serem responsáveis por grande parte dos gastos com recursos humanos, materiais e tecnologia no setor de Saúde ${ }^{1,2}$. Neste contexto, o Sistema de Informações Hospitalares do Sistema Único de Saúde (SIH-SUS) vem se configurando como um dos principais instrumentos tanto para a implantação e avaliação das políticas relacionadas à assistência médico-hospitalar, no que diz respeito à sua organização e financiamento no sistema público de saúde, quanto para fins epidemiológicos ${ }^{3}$.

No Brasil, apesar do crescente uso dos sistemas de informações em pesquisas sobre morbidades e custos registrados nas autorizações de internação hospitalar (AIHs), são poucos os estudos sobre as internações por transtornos mentais e comportamentais, embora se saiba que elas implicam em alto custo financeiro para assistência em saúde. Além disso, segundo a Organização Mundial de Saúde (OMS), a prevalência dos transtornos mentais na população mundial está em torno de $10 \%$, superando $25 \%$ quando considerados episódios durante toda a vida ${ }^{4}$. Adicionalmente à alta frequência com que ocorrem, esses transtornos lideram, ainda, o ranking das doenças listadas como as principais causas de anos de vida vividos com incapacidade (Years Lived with Disability YLDs), ultrapassando, inclusive, as doenças cardiovasculares e HIV/AIDS ${ }^{4-6}$.

Embora ainda haja questionamentos dos pesquisadores da área da Saúde Mental quanto à confiabilidade acerca dos registros relacionados aos transtornos mentais nos sistemas de informação em saúde brasileiros, estudos recentes vêm apontando o seu uso como um meio simplificado para a obtenção de dados sobre a situação dos transtornos mentais e comportamentais, permitindo aos profissionais formular e executar ações importantes na área ${ }^{7-9}$. Os registros das internações hospitalares em psiquiatria do SIH-SUS são mais utilizados nessas pesquisas que os dados presentes no Sistema de Informação Ambulatorial (SIA). Isso porque são considerados de menor confiabilidade para os diagnósticos contemplados pelo capítulo V da CID-10 (transtornos mentais e comportamentais da Classificação Internacional de Doenças $-10^{\mathrm{a}}$ revisão) ${ }^{8}$, embora a assistência ambulatorial em psiquiatria encontre-se em processo de expansão com a reforma psiquiátrica e a política de desinstitucionalização dos pacientes, sancionada pela Lei $\mathrm{n}^{\circ} 10.216 \mathrm{em} 2001^{7}$.

O SIH-SUS é alimentado pelos dados da AIH, que se referem à morbidade e mortalidade, além de informações financeiras sobre o atendimento prestado ${ }^{3}$. O diagnóstico psiquiátrico presente no laudo para solicitação da $\mathrm{AIH}$ é fornecido pelo médico psiquiatra no momento da internação do paciente. Ele é composto pelo diagnóstico principal, pelo secundário e causas associadas, definidos segundo os critérios da CID-10.

Os diagnósticos psiquiátricos mais frequentes nas bases do SIH-SUS são referentes a transtornos mentais severos e persistentes, tais como os psicóticos, cuja terapêutica muitas vezes requer internação ${ }^{8}$. Apesar das críticas em relação à baixa confiabilidade para algumas categorias diagnósticas, sobretudo quando comparadas internações por esquizofrenia de um mesmo indivíduo em hospitais diferentes ${ }^{10}$, o diagnóstico em psiquiatria vem se aprimorando consideravelmente nos últimos anos, principalmente a partir da formulação dos critérios diagnósticos utilizados pelo Diagnostic and Statistical Manual of Mental Disorders III (DSM-III) na década de 1980, que aumentou a confiabilidade da classificação psiquiátrica ${ }^{11}$.

Da mesma forma, pode-se afirmar que o uso dos sistemas de informações em saúde em pesquisas vem apresentando notável expansão a partir das décadas de 1980 e 1990, principalmente dos sistemas de informação sobre mortalidade, morbidade hospitalar e nascidos vivos ${ }^{1}$. A má qualidade das informações, outrora presente nos sistemas de informação devido a falhas na cobertura, falta de padronização e normatização dos sistemas e dificuldades de compatibilizar as informações e o acesso a elas, agora vem sendo superada graças a ações desenvolvidas pelo Ministério da Saúde, tais como a padronização dos instrumentos básicos de coleta de dados ${ }^{12}$ e a criação, em 1996, da Rede Interagencial de Informações para a Saúde (RIPSA), em cooperação com a Organização Pan-Americana da Saúde (OPAS). A RIPSA surge como instância qualificadora de informações para produzi-las e disponibilizá-las no contexto das relações entre as três esferas do SUS, outros setores de governo e as entidades de ensino e pesquisa ${ }^{13}$.

Assim, o presente estudo vem reforçar a possibilidade de uso desses sistemas de informação no campo da saúde mental, tendo como objetivo analisar o perfil das internações psiquiátricas no estado do Rio de Janeiro ao longo do período de 1999 a 2010, bem como as repercussões da nova política de saúde mental na assistência hospitalar. Com isso, esse artigo visa contribuir para o conhecimento sobre os transtornos mentais, em especial os severos e persistentes, incluindo os do espectro esquizofrênico, que se destacam pela gravidade do comprometimento psíquico, pela carga de doença provocada na vida dos indivíduos acometidos e pela cronicidade e necessidade de cuidados contínuos e, inclusive, de internações, em determinados casos. 


\section{METODOLOGIA}

Trata-se de um estudo descritivo sobre o perfil das internações psiquiátricas presentes na base de dados do SIH-SUS no período de janeiro de 1999 a dezembro de 2010 no Estado do Rio de Janeiro.

Os arquivos reduzidos de AIH no formato "dbc", de acesso livre e com registros correspondentes a cada AIH paga por unidade da federação e período, foram obtidos por meio do SIH-SUS no endereço eletrônico do Departamento de Informática do SUS - DATASUS (http://www.datasus.gov.br). O programa Tab para Windows (TabWin) foi utilizado na tabulação e análise descritiva dos dados contidos nas bases do SIH-SUS.

A variável "internações", utilizada neste estudo, constitui um valor aproximado das internações, fornecido pelo próprio DATASUS, que diz respeito à quantidade de AIHs pagas no período, não considerando as de prorrogação (longa permanência), muito frequentes quando se tratam de internações psiquiátricas. A partir da análise dessa informação, foi realizada uma série temporal, com a evolução da estimativa do número de internações ao longo dos anos de 1999 a 2010.

Os dados de interesse analisados foram: sexo, faixa etária (10 a 14 anos, 15 a 19 anos, 20 a 29 anos, 30 a 39 anos, 40 a 49 anos, 50 a 59 anos, $\geq 60$ anos e idade ignorada), especialidade (Clínica cirúrgica, Obstetrícia, Clínica médica, Cuidados prolongados, Tisiologia, Pediatria, Reabilitação e não discriminado), grupos de doenças (capítulos da CID-10), transtornos mentais (lista de morbidades da CID-10), tipo de hospital (psiquiátrico, geral e especializado) e valor total. As variáveis "grupos de doenças" e "transtornos mentais" são provenientes do preenchimento da causa da internação no laudo de solicitação da AIH, ou seja, do diagnóstico principal fornecido pelo médico no momento da internação, segundo a Classificação Internacional de Doenças, na sua $10^{\mathrm{a}}$ revisão.

A variável "valor total" corresponde ao valor referente às AIHs pagas no período. Vale destacar que não obrigatoriamente ele corresponde ao total repassado ao estabelecimento, pois, dependendo da situação das unidades, estas recebem recursos orçamentários ou pode haver retenções e pagamentos de incentivos não apresentados. Portanto, deve ser considerado o valor aprovado da produção.

A série histórica (1999-2010) foi obtida pelo total das frequências absolutas das internações de cada triênio: 1999 a 2001, 2002 a 2004, 2005 a 2007 e 2008 a 2010. Esta abordagem possibilita melhor visualização e análise dos dados quando se trabalha com longos períodos de estudo, sem prejudicar, no entanto, a observação das variações ocorridas no decorrer do mesmo. Também foram analisadas as frequências relativas para cada variável estudada.
A pesquisa utilizou dados secundários disponíveis em sistemas públicos de informações, não sendo necessária a submissão do projeto ao Comitê de Ética.

\section{RESULTADOS}

De acordo com o Gráfico 1, o número de internações por transtornos mentais e comportamentais ao longo do período estudado (1999 a 2010) apresentou tendência decrescente, apesar de serem observadas pequenas flutuações entre alguns anos. Em 1999, observou-se total de 60.494 internações por transtornos mentais no estado do Rio de Janeiro, enquanto no ano de 2010 esse número foi reduzido para 17.826 internações, ou seja, houve queda de $70 \%$. A maior diminuição do período foi verificada a partir de 2001, no qual houve redução de mais de 18 mil internações já para o ano seguinte (2002). A frequência relativa das internações foi avaliada quanto ao sexo, faixa etária e diagnóstico por triênios no período de 1999 a 2010, revelando predomínio das internações por transtornos mentais em pacientes do sexo masculino (cerca de 65\%). Além disso, essas internações foram mais frequentes nas faixas etárias de 30 a 39 anos e 40 a 49 anos para ambos os sexos, em todos os triênios analisados (Tabela 1). Com relação aos diagnósticos presentes nas AIHs, a esquizofrenia e outros transtornos esquizotípicos e delirantes foram responsáveis pelo maior percentual (50 a $60 \%$ ) das internações por transtornos mentais durante todos os triênios estudados, para ambos os sexos e todas as faixas etárias. Dentre os pacientes internados por esses motivos, cerca de $60 \%$ eram homens.

As internações por esquizofrenia foram mais frequentes na faixa etária dos 20 a 30 anos para os pacientes do sexo masculino, enquanto para os do feminino essa frequência foi maior na faixa dos 30 a 39 anos.

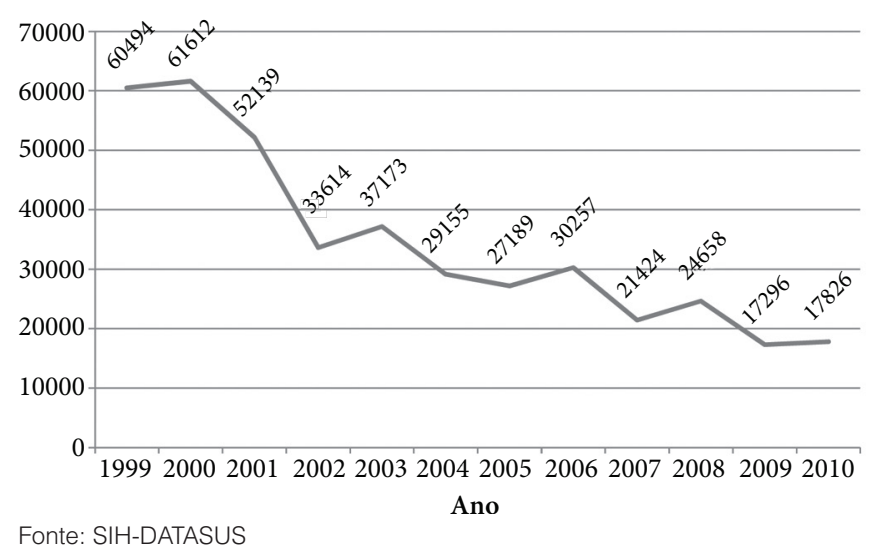

Gráfico 1. Total de internações por transtornos mentais e comportamentais (capítulo V da CID-10) ocorridas durante o período de 1999 a 2010 no estado do Rio de Janeiro 


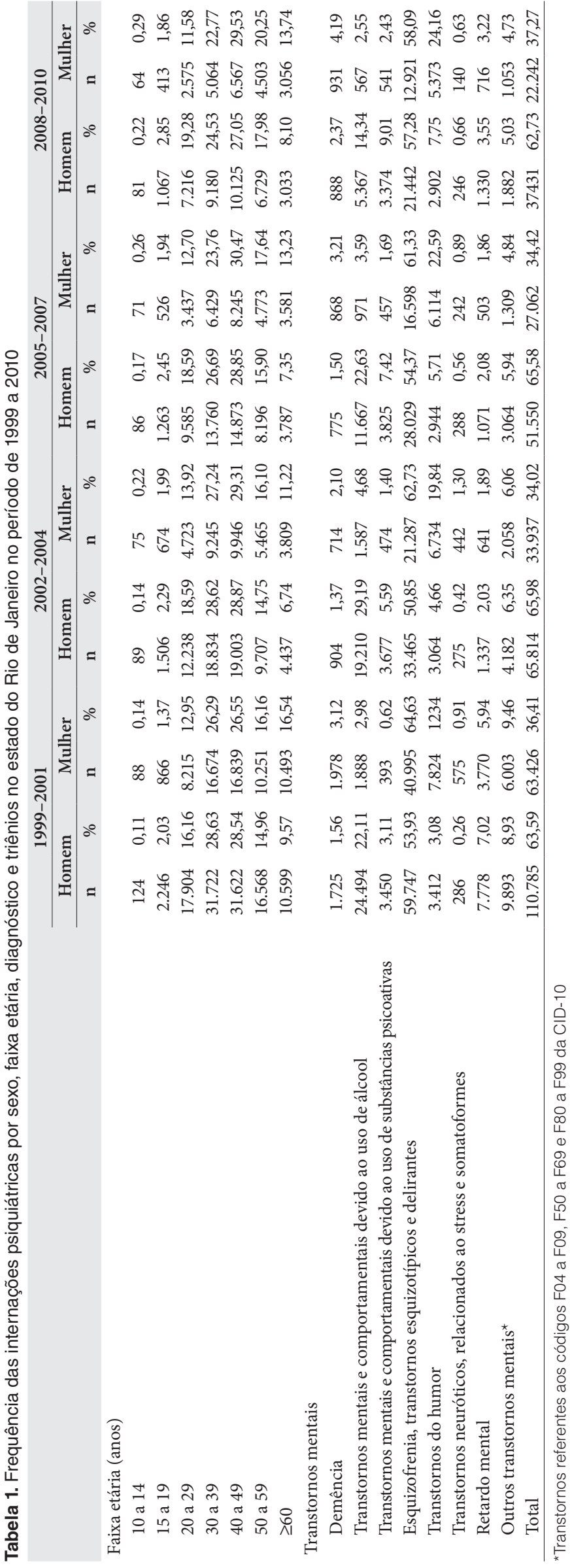

Posteriores à esquizofrenia, os diagnósticos mais frequentes nas AIHs foram os transtornos relacionados ao uso de álcool e os de humor. No entanto, observa-se diferença na segunda causa de internações psiquiátricas em relação ao sexo. Para os homens, o segundo diagnóstico mais frequente nas internações por transtornos mentais foram os relacionados ao abuso e dependência de álcool, enquanto para as mulheres foram os de humor.

As internações por transtornos relacionados ao uso de substâncias foram muito frequentes na população jovem ao longo de todos os triênios, sendo alto o número de hospitalizações por transtornos relacionados ao uso de substâncias psicoativas na faixa dos 20 aos 29 anos, enquanto os pacientes internados por transtornos relacionados ao uso de álcool tinham, em média, 30 a 49 anos.

Os transtornos do humor apresentaram ligeiro aumento percentual durante o período, sendo responsáveis por quase um quarto das internações psiquiátricas em mulheres no último triênio, de 2008 a 2010 (Tabela 1). Vale destacar que, dentre os transtornos de humor, a principal causa de internação em ambos os sexos durante todo o período foram os transtornos bipolares (cerca de 60\%), seguidos pelos depressivos (aproximadamente 20\%) e episódios maníacos (em torno de $10 \%)$. Com relação às faixas etárias mais frequentes nas internações por esses transtornos em mulheres, destacam-se o intervalo dos 20 aos 49 anos e a faixa dos 50 a 59 anos.

Quando analisadas as internações por todas as especialidades, observou-se que as referentes à especialidade psiquiatria corresponderam a mais de $10 \%$ do total durante todos os triênios, incluindo-se a clínica cirúrgica, obstetrícia, clínica médica, cuidados prolongados (crônicos), tisiologia, pediatria e reabilitação (Tabela 2).

Com relação ao valor total referente às AIHs pagas no período, no triênio de 1999 a 2001 as internações por transtornos mentais e comportamentais (Capítulo V da CID-10) representaram $21,9 \%$ do valor total gasto, liderando o ranking dos grupos de doenças responsáveis pelos maiores gastos com internações (Tabela 3). Nos demais triênios analisados (20022004, 2005-2007, 2008-2010), observou-se tendência decrescente nos gastos com internações por transtornos mentais. No entanto, esse grupo continuou representando um dos maiores gastos com internações, perdendo apenas para as doenças do aparelho circulatório (Capítulo IX da CID-10).

No que se refere ao tipo de hospital onde ocorreram as internações, o psiquiátrico ainda responde por mais de $90 \%$ nos dois triênios estudados (Tabela 4). Observa-se, ainda, pequeno aumento, de cerca de $2 \%$, das internações por transtornos mentais em hospitais gerais enquanto há discreta tendência à redução no total das internações por esses transtornos em hospitais psiquiátricos entre os triênios analisados. 
Tabela 2. Frequência total e relativa de internações segundo especialidade por triênios no estado do Rio de Janeiro no período de 1999 a 2010

\begin{tabular}{|c|c|c|c|c|c|c|c|c|c|c|}
\hline \multirow{2}{*}{ Especialidade } & \multicolumn{2}{|c|}{ 1999-2001 } & \multicolumn{2}{|c|}{ 2002-2004 } & \multicolumn{2}{|c|}{ 2005-2007 } & \multicolumn{2}{|c|}{ 2008-2010 } & \multicolumn{2}{|c|}{ Total } \\
\hline & n & $\%$ & $\mathrm{n}$ & $\%$ & $\mathbf{n}$ & $\%$ & $\mathrm{n}$ & $\%$ & $\mathrm{n}$ & $\%$ \\
\hline Psiquiatria & 292.886 & 10,06 & 364.819 & 13,76 & 298.784 & 12,38 & 223.054 & 10,36 & 1.179 .543 & 11,64 \\
\hline Outras especialidades ${ }^{*}$ & 2.619 .657 & 89,94 & 2.286 .313 & 86,24 & 2.114 .687 & 87,62 & 1.930 .627 & 89,64 & 8.951 .284 & 88,36 \\
\hline Total & 2.912 .543 & 100,00 & 2.651 .132 & 100,00 & 2.413 .471 & 100,00 & 2.153 .681 & 100,00 & 10.130 .827 & 100,00 \\
\hline
\end{tabular}

*Clínica cirúrgica, obstetrícia, clínica médica, cuidados prolongados (crônicos), tisiologia, pediatria e reabilitação

Tabela 3. Valor total referente às autorizações de internação hospitalares pagas por capítulo da CID-10 e triênios no estado do Rio de Janeiro no período de 1999 a 2010

\begin{tabular}{|c|c|c|c|c|c|c|c|c|}
\hline \multirow{2}{*}{ Capítulo da CID-10 } & \multicolumn{2}{|c|}{ 1999-2001 } & \multicolumn{2}{|c|}{ 2002-2004 } & \multicolumn{2}{|c|}{ 2005-2007 } & \multicolumn{2}{|c|}{ 2008-2010 } \\
\hline & $\mathbf{n}$ & $\%$ & $\mathbf{n}$ & $\%$ & $\mathbf{n}$ & $\%$ & $\mathbf{n}$ & $\%$ \\
\hline I. Algumas doenças infecciosas e parasitárias & 40.191.019,95 & 3,50 & $46.374 .965,03$ & 3,68 & $47.428 .142,74$ & 3,48 & $78.432 .935,38$ & 4,93 \\
\hline II. Neoplasias (tumores) & $79.385 .460,46$ & 6,92 & $98.673 .865,04$ & 7,82 & $106.270 .294,75$ & 7,8 & $127.326 .563,31$ & 8,01 \\
\hline $\begin{array}{l}\text { III. Doenças sangue órgãos hematopoéticos } \\
\text { e transtimunitários }\end{array}$ & $6.056 .588,32$ & 0,53 & $6.348 .074,64$ & 0,50 & $5.792 .406,78$ & 0,42 & $6.570 .492,28$ & 0,41 \\
\hline $\begin{array}{l}\text { IV. Doenças endócrinas nutricionais e } \\
\text { metabólicas }\end{array}$ & $2.2175 .768,2$ & 1,93 & $23.519 .717,57$ & 1,86 & $25.122 .572,92$ & 1,84 & $28.228 .295,12$ & 1,77 \\
\hline V. Transtornos mentais e comportamentais & $251.263 .000,3$ & 21,90 & $251.259 .459,58$ & 19,92 & $237.275 .646,60$ & 17,41 & $229.031 .479,39$ & 14,40 \\
\hline VI. Doenças do sistema nervoso & $37.332 .550,6$ & 3,25 & $46.027 .957,37$ & 3,65 & 49.827.212,02 & 3,66 & $60.216 .802,64$ & 3,79 \\
\hline VII. Doenças do olho e anexos & $11.615 .064,65$ & 1,01 & $6.937 .564,64$ & 0,55 & $8.051 .251,59$ & 0,59 & $9.460 .515,27$ & 0,59 \\
\hline $\begin{array}{l}\text { VIII. Doenças do ouvido e da apófise } \\
\text { mastoide }\end{array}$ & $817.319,63$ & 0,07 & $729.013,89$ & 0,06 & $782.981,67$ & 0,06 & $1.163 .878,11$ & 0,07 \\
\hline IX. Doenças do aparelho circulatório & $231.062 .778,01$ & 20,14 & $279.357 .976,57$ & 22,14 & $325.730 .300,49$ & 23,89 & $401.595 .591,07$ & 25,25 \\
\hline X. Doenças do aparelho respiratório & $64.555 .866,32$ & 5,63 & $64.807 .747,21$ & 5,14 & $71.864 .567,64$ & 5,27 & 104.778.775,48 & 6,59 \\
\hline XI. Doenças do aparelho digestivo & 63890806.74 & 5,57 & $76.947 .016,13$ & 6,10 & $83.906 .246,75$ & 6,16 & $98.286 .239,41$ & 6,18 \\
\hline XII. Doenças da pele e do tecido subcutâneo & $7.075 .561,15$ & 0,62 & $8.874 .135,72$ & 0,70 & $9.011 .154,84$ & 0,66 & $11.788 .969,90$ & 0,74 \\
\hline $\begin{array}{l}\text { XIII. Doenças do sistema osteomuscular e } \\
\text { tecido conjuntivo }\end{array}$ & 24.659.286,17 & 2,15 & $32.192 .988,30$ & 2,55 & $38.279 .753,65$ & 2,81 & $37.092 .924,76$ & 2,33 \\
\hline XIV. Doenças do aparelho geniturinário & $41.207 .130,00$ & 3,59 & $48.782 .831,99$ & 3,87 & $46.610 .245,52$ & 3,42 & $58.454 .464,76$ & 3,68 \\
\hline XV. Gravidez, parto e puerpério & $160.216 .498,31$ & 13,96 & $156.891 .242,74$ & 12,44 & $172.315 .903,74$ & 12,64 & 194.829.366,55 & 12,25 \\
\hline $\begin{array}{l}\text { XVI. Algumas afecções originadas no } \\
\text { período perinatal }\end{array}$ & $253.665,32$ & 0,02 & $49.647,93$ & 0,01 & $105.391,28$ & 0,01 & $613.558,08$ & 0,04 \\
\hline $\begin{array}{l}\text { XVII. Malformações congênitas, } \\
\text { deformidades e anomalias cromossômicas }\end{array}$ & $6.570 .978,29$ & 0,57 & $6.517 .424,87$ & 0,52 & $8.843 .623,56$ & 0,65 & $12.264 .109,92$ & 0,77 \\
\hline $\begin{array}{l}\text { XVIII. Sintomas, sinais e achados anormais } \\
\text { de exames clínicos e de laboratório, não } \\
\text { classificados em outra parte }\end{array}$ & $5.204 .545,33$ & 0,45 & $6.835 .636,07$ & 0,54 & $8.766 .296,54$ & 0,64 & $10.508 .914,09$ & 0,66 \\
\hline $\begin{array}{l}\text { XIX. Lesões, envenenamento e algumas } \\
\text { outras consequências de causas externas }\end{array}$ & 70532914.32 & 6,15 & $88.294 .559,39$ & 7,00 & 105.761.601,06 & 7,76 & $111.515 .104,02$ & 7,01 \\
\hline $\begin{array}{l}\text { XX. Causas externas de morbidade e } \\
\text { mortalidade }\end{array}$ & $6.543 .933,39$ & 0,57 & $1.165 .800,44$ & 0,09 & $244.349,33$ & 0,02 & $686.354,77$ & 0,04 \\
\hline XXI. Contatos com serviços de saúde & $16.646 .356,58$ & 1,45 & 10.967.472,92 & 0,87 & 10.112.388,90 & 0,74 & $7.583 .823,25$ & 0,48 \\
\hline $\begin{array}{l}\text { CID } 10^{\mathrm{a}} \text { Revisão não disponível ou } \\
\text { não preenchido }\end{array}$ & $45.191,40$ & 0,01 & - & - & $1.092 .265,83$ & 0,08 & - & - \\
\hline Total & $1.147 .302 .283,45$ & 100,00 & $1.261 .555 .098,04$ & 100,00 & $1.363 .194 .598,20$ & 100,00 & $1.590 .429 .157,56$ & 100,00 \\
\hline
\end{tabular}


Tabela 4. Frequência das internações por transtornos mentais e comportamentais por tipo de hospital e triênios no estado do Rio de Janeiro no período de 2005 a 2010

\begin{tabular}{lcccc}
\multirow{2}{*}{ Tipo de hospital* } & \multicolumn{2}{c}{$\mathbf{2 0 0 5}-\mathbf{2 0 0 7}$} & \multicolumn{2}{c}{$\mathbf{2 0 0 8}-\mathbf{2 0 1 0}$} \\
\cline { 2 - 5 } & $\mathbf{n}$ & $\mathbf{\%}$ & $\mathbf{n}$ & $\mathbf{\%}$ \\
Geral & 15.435 & 5,23 & 16.759 & 7,25 \\
Psiquiátrico & 271.732 & 92,16 & 212.546 & 91,92 \\
Especializado & 7.679 & 2,60 & 1.934 & 0,84 \\
Total & 294.846 & 100,00 & 231.239 & 100,00 \\
\hline
\end{tabular}

*Informações disponibilizadas somente a partir de agosto de 2005

\section{DISCUSSÃO}

Os resultados do presente estudo revelam a crescente redução do número de internações por transtornos mentais e comportamentais no SUS nos últimos anos, principalmente a partir de 2001. Tal tendência reflete o movimento pela desinstitucionalização dos pacientes com transtornos mentais, que, embora tenha começado a se desenvolver no campo das políticas de saúde do Brasil já na década de $1980^{14,15}$, somente foi sancionado pela Lei 10.216, que dispõe sobre a proteção e os direitos das pessoas portadoras de transtornos mentais e redireciona o modelo assistencial em saúde mental ${ }^{16}$, em 2001. Essa nova política de saúde mental, baseada na reforma da assistência psiquiátrica, prevê a redução progressiva de leitos e a reinserção social dos pacientes por meio da implantação de uma rede de serviços extra-hospitalares.

Dessa forma, a redução do número de leitos psiquiátricos deve ser necessariamente acompanhada por políticas e serviços que permitam a reintegração dos internos aos lares de seus familiares e à sociedade, tais como a implantação do Programa De Volta Para Casa, a criação de residências terapêuticas, a expansão dos Centros de Atenção Psicossocial (CAPS), a inclusão de ações de saúde mental na atenção básica pelo Programa de Saúde da Família (PSF) e o credenciamento de leitos psiquiátricos em hospitais gerais ${ }^{17}$. No entanto, um relatório do Tribunal de Contas da União sobre a avaliação da atenção em saúde mental, publicado em 2005, destaca a morosidade do processo de desinstitucionalização em curso, mostrando que os hospitais psiquiátricos no Brasil continuam responsáveis pelas internações desses pacientes, mantendo, ainda, a sua característica asilar ${ }^{18}$.

Vários estudos nacionais vêm comprovando a lentidão na redução de leitos psiquiátricos, bem como a insuficiente expansão dos serviços extra-hospitalares ${ }^{7,19-21}$. Em nossa pesquisa, observamos que o hospital psiquiátrico ainda é responsável pela maior parte das internações por transtornos mentais e comportamentais no estado do Rio de Janeiro, tendo a proporção dessas internações em hospitais gerais aumentado apenas de 5 para $7 \%$ entre os triênios de 2005 a 2007 e 2008 a 2010. Esse número, embora semelhante à média nacional, que é de cerca de $4 \%^{22}$, está aquém da proporção encontrada em alguns países da América Latina, como Colômbia (48,8\%), Venezuela (26,8\%), Chile (13,8\%) e Uruguai $(11,2 \%)^{23}$, e, ainda, em estados da região Sul do Brasil, como o Rio Grande do Sul, em torno de 30\%, talvez devido ao pioneirismo legislativo do local em relação à reforma da assistência psiquiátrica ${ }^{8}$.

Cabe destacar que a implementação de leitos destinados a pacientes com transtornos mentais em hospitais gerais constitui uma ação estratégica para a redução do estigma da doença mental, bem como para a melhoria do acesso ao serviço e do intercâmbio com outras especialidades médicas, assegurando melhor atenção à saúde física dessas pessoas ${ }^{24}$.

Além de indicada como último recurso terapêutico e ser considerada pouco eficiente e excludente, a internação psiquiátrica, especialmente a de caráter asilar, com longa duração, implica em altos gastos para a saúde pública. Os dados do presente estudo revelaram que elas foram responsáveis por mais de $20 \%$ do valor total de todas as internações, por todos os grupos de doenças no estado do Rio de Janeiro, superando, inclusive, as hospitalizações por doenças do aparelho circulatório no primeiro triênio analisado (1999 a 2001). Embora nos períodos seguintes esses gastos tenham decrescido, até mesmo devido à diminuição já observada na frequência de internações por transtornos mentais, as essas internações continuaram ocupando o segundo lugar no grupo de doenças que apresentaram maiores gastos com internações, representando custo de mais de $\mathrm{R} \$ 229$ milhões no último triênio de análise (2008 a 2010). Segundo uma pesquisa que analisou os gastos nacionais com internações de pacientes com transtornos mentais no ano de 2004, o valor pago pelo SUS atingiu R $\$ 487$ milhões, sendo o Rio de Janeiro responsável por mais de $17 \%$ desse total, perdendo apenas para São Paulo $(30 \%)^{7}$.

O orçamento do SUS em saúde mental tem mostrado constante inversão do financiamento: cada vez mais se investe na rede substitutiva dos serviços em detrimento dos hospitais psiquiátricos. Já o gasto federal com as internações nesta mesma categoria de hospitais foi de $29,44 \%$ em 2010, enquanto o valor dispendido em ações e serviços extra-hospitalares representou $70,56 \%$ do total ${ }^{25}$. Contudo, a discussão sobre a lógica da produtividade que ainda sustenta a alocação de recursos do SUS aos equipamentos substitutivos permanece atual. Observa-se que a remuneração está vinculada ao episódio de doença por meio de procedimentos (ambulatorial ou hospitalar) ou diárias hospitalares. Portanto, apesar da inversão do financiamento em saúde mental, permanece a necessidade da análise da estrutura desse financiamento em relação ao modelo assistencial que se deseja ${ }^{26}$. O Relatório Final da 
IV Conferência Nacional de Saúde Mental - Intersetorial ${ }^{27}$ propõe que, além dos recursos financeiros à Saúde Mental, seja garantida pelas três esferas do governo a regulamentação desses recursos, que devem atender modificações na forma de pagamento e na atribuição de rubrica orçamentária específica, entre outros, levando à extinção do critério de pagamento por produção assistencial.

Os transtornos mentais ainda vêm sendo mundialmente reconhecidos pela sua importância clínica e epidemiológica. A OMS alerta que a cada quatro pessoas uma sofre ao menos um transtorno mental em algum momento de sua vida ${ }^{4}$. Transtornos como a esquizofrenia, a depressão, os relacionados ao uso de substâncias e a demência lideram as principais causas de anos de vida vividos com incapacidade, ultrapassando, inclusive, o câncer e as doenças cardiovasculares ${ }^{4-6}$. Em nossos dados, observamos que os três primeiros transtornos foram responsáveis pela maior parte das internações por transtornos mentais no estado do Rio de Janeiro em todos os triênios avaliados.

Conforme verificado no presente estudo, os transtornos mentais e comportamentais corresponderam a mais de $10 \%$ do total de internações por todas as especialidades durante todos os triênios analisados, incluindo a clínica cirúrgica, obstetrícia, clínica médica, cuidados prolongados (crônicos), tisiologia, pediatria e reabilitação.

Apesar da relevância do tema, ainda é observado que os transtornos mentais não recebem a merecida atenção das pesquisas no campo da saúde pública. Nesse sentido, ainda pouco se sabe sobre o perfil dos pacientes internados, especialmente os provenientes de hospitais psiquiátricos, e essa informação é de fundamental importância para a implementação das mudanças na política assistencial em saúde mental.

Em nosso estudo, de uma maneira geral, as internações psiquiátricas foram mais frequentes nas faixas etárias de 30 a 39 anos e 40 a 49 anos para ambos os sexos, em todos os triênios analisados. O diagnóstico mais verificado nas AIHs foi a esquizofrenia e outros transtornos esquizotípicos e delirantes, responsáveis por até $60 \%$ das internações por transtornos mentais durante todos os triênios estudados, para ambos os sexos e todas as faixas etárias.

Apesar da sua baixa prevalência na população, em torno de 0,5 a $1,5 \%$, não havendo evidência de diferenças entre os $\operatorname{sexos}^{28,29}$, a esquizofrenia é historicamente o transtorno responsável pela maior parte das admissões hospitalares em psiquiatria ${ }^{29}$. Exceto na presença de história familiar positiva para transtornos psicóticos em parentes de primeiro grau, a literatura aponta que a idade na primeira admissão hospitalar costuma ser mais precoce para os homens (média de 25 anos) do que para as mulheres (média de 30 anos) ${ }^{28,29}$.
O mesmo é observado em nossos dados, nos quais as internações por esquizofrenia foram mais frequentes na faixa etária dos 20 a 30 anos para os pacientes do sexo masculino, enquanto para os do sexo feminino essa presença foi maior na faixa dos 30 a 39 anos.

Dentre os pacientes internados por esquizofrenia e outros transtornos esquizotípicos e delirantes ao longo de todo o período estudado, cerca de $60 \%$ eram homens, assim como encontrado em censos realizados com internos de hospitais psiquiátricos do Rio de Janeiro ${ }^{30,31}$. Apesar de a literatura não apontar diferenças marcantes entre os sexos para esse transtorno, segundo os autores de um censo realizado em 20 hospitais psiquiátricos da cidade do Rio de Janeiro, uma possível explicação para tal divergência encontrada entre os gêneros pode ser o fato de haver maior probabilidade de homens serem internados na presença de algum transtorno psicótico em comparação com as mulheres ou, ainda, o alcoolismo e a psicose alcoólica apresentarem-se mais prevalentes entre os homens ${ }^{31}$.

As internações por transtornos mentais foram mais frequentes em pacientes do sexo masculino, principalmente quando nos referimos às internações por transtornos relacionados ao uso do álcool e de substâncias psicoativas, já apontados pela literatura como mais frequentes em homens, especialmente jovens ${ }^{5,28,32}$. Em nosso estudo, esse tipo de internação também foi mais frequente na população jovem, sendo as por transtornos relacionados ao uso de substâncias psicoativas muito frequentes na faixa dos 20 aos 29 anos, enquanto os pacientes internados por transtornos relacionados ao uso de álcool tinham em média 30 a 49 anos.

De acordo com a OMS, no ranking das 20 doenças que acarretam anos de vida vividos com alguma incapacidade entre homens de 15 a 44 anos, os transtornos devido ao uso de álcool assumem o segundo lugar e os devido ao uso de outras drogas encontram-se na nona posição ${ }^{4,5}$. Diversas doenças físicas, mentais e até mesmo a mortalidade por causas externas estão associadas ao uso abusivo do álcool e outras substâncias psicoativas, tais como acidentes fatais de trânsito, homicídios, suicídios e comportamentos violentos. Dessa forma, esses transtornos geram não só um significativo custo para o setor de saúde como também para toda a sociedade.

Embora a atual política pública nacional de atenção a usuários de álcool e drogas seja dirigida à assistência em equipamentos de saúde extra-hospitalares e de menor complexidade, como os Centros de Atenção Psicossocial - Álcool e outras drogas (CAPS AD) ${ }^{33}$, um estudo revelou que são crescentes os gastos com internações por transtornos relacionados ao uso de substâncias no Brasil, chegando a mais de 171 milhões no período de 2002 a $2004^{34}$. 
Em contrapartida, enquanto as internações por uso de substâncias foram mais frequentes em homens, as internações por transtornos do humor, em especial o bipolar e a depressão, foram mais frequentes em mulheres, o que também vem ao encontro das estatísticas apresentadas pelas pesquisas epidemiológicas da área ${ }^{28,35-37}$. Tais internações são mais presentes em mulheres em idade reprodutiva, especialmente na faixa dos 20 aos 49 anos de idade.

Sabe-se que o período gravídico-puerperal é apontado pela literatura científica como a fase de maior frequência de transtornos mentais na mulher, sendo a depressão o de maior prevalência nesse período ${ }^{35-37}$. Estima-se que aproximadamente uma em cada cinco mulheres apresenta episódios depressivos durante a gravidez e pós-parto ${ }^{38}$, períodos que envolvem inúmeras alterações físicas, hormonais, psíquicas e de inserção social na vida, podendo, portanto, ter reflexos na saúde mental.

Outra faixa etária que vem se destacando nas internações em mulheres por transtornos de humor é a de 50 a 59 anos, o que pode estar associado também a alterações hormonais do período de menopausa, que, por sua vez, é apontada como fase de risco para esses transtornos, com destaque para a depressão $0^{36,37}$.

Epidemiologicamente, a depressão é mais frequente que o transtorno bipolar, com prevalências durante a vida que variam de 10 a $25 \%$ para mulheres e 5 a $12 \%$ para os homens, contra frequências de 0,4 a 1,6\% para o transtorno bipolar ${ }^{28}$. No entanto, em nosso estudo, este transtorno apareceu em primeiro lugar dentre as causas de internações por transtornos do humor, provavelmente devido à possibilidade de sua terapêutica requerer internações eventuais, especialmente nos casos que apresentaram sintomas psicóticos e episódios depressivos maiores, enquanto o transtorno depressivo, muito presente em sua forma leve e moderada, na maioria dos casos não necessita de internações.

Ainda sobre os transtornos de humor, vale destacar que as internações tendo eles como motivo apresentaram aumento percentual durante o período analisado, sendo responsáveis por quase uma em cada quarto internações psiquiátricas em mulheres no triênio de 2008 a 2010. Esse aumento também tem sido observado em outros estudos, inclusive internacionais, e contribui para a redução proporcional nas internações por esquizofrenia ${ }^{8,9,32,39,40}$. O fato pode ser decorrente, ainda, de um viés de diagnóstico, já que muitas vezes é orientado pelas possibilidades de tratamento, como, por exemplo, o aumento observado na proporção do diagnóstico de transtornos afetivos na década de 1970, após a introdução do lítio ${ }^{41}$.
Apesar de seu caráter inovador quanto ao uso do SIH em pesquisas na área da saúde mental, considerada a existência de poucos estudos nacionais sobre o tema, a presente pesquisa apresenta algumas limitações metodológicas. Em termos de análise da morbidade, por terem sido usados apenas os dados do SIH, os transtornos aqui analisados podem ser considerados, quanto à sua gravidade, de moderados a graves, visto que demandaram a necessidade de internações, não sendo contemplados aqui os transtornos mentais leves, muito frequentes em termos epidemiológicos.

Além disso, embora a variável "internações", utilizada neste estudo, fosse um valor aproximado das internações fornecido pelo próprio DATASUS, já que não incluía as AIHs de prorrogação (longa permanência), muito comuns em internações psiquiátricas, as transferências e reinternações poderiam estar computadas nessa variável, de modo que o número encontrado possa ter sido ligeiramente superestimado. Vale ressaltar ainda que foram apenas analisadas as internações nas unidades hospitalares participantes do SUS (públicas ou particulares conveniadas), não estando incluídas nas análises as ocorridas na rede privada de saúde.

Assim como em qualquer pesquisa que utilize dados secundários de Sistemas de Informação em Saúde, também não podemos afastar a possibilidade de erros de preenchimento e digitação nos bancos utilizados. Entretanto, diversos estudos apontam que os SIS no Brasil vêm apresentando melhoras progressivas quanto à qualidade de seus dados ${ }^{1,2,8,9}$, até mesmo em decorrência de esforços empreendidos pelo Ministério da Saúde em parceria com outros órgãos, que incluíram ações como a padronização dos instrumentos básicos de coleta de dados $^{12}$ e a criação da RIPSA ${ }^{13}$.

Dessa forma, este artigo reforça a possibilidade de utilização dos SIS em pesquisas sobre morbidade na área da saúde mental, que, embora já muito frequente em estudos internacionais ${ }^{39,42-45}$, ainda conta com uma tímida produção bibliográfica nacional ${ }^{7,8,46}$. Conhecer o perfil das internações por transtornos mentais pelo Sistema de Informações Hospitalares pode ser útil não só para o conhecimento epidemiológico desses transtornos, mas também para avaliar a efetividade das políticas públicas implementadas na área da saúde mental, bem como para o planejamento de novas ações de saúde. O uso de dados secundários em pesquisas, além do baixo custo gerado, constitui importante fonte de informações epidemiológicas, principalmente em países com cobertura universal dos serviços públicos de saúde como o Brasil, no qual cerca de $70 \%$ da população é usuária do SUS ${ }^{47}$, porém novos estudos são necessários para que sejam agregadas informações sobre as internações psiquiátricas na rede privada de saúde. 


\section{REFERÊNCIAS}

1. Bittencourt SA, Camacho LAB, Leal MC. O Sistema de Informação Hospitalar e sua aplicação na saúde coletiva. Cad Saúde Pública. 2006;22(1):19-30

2. Mello Jorge MHP, Gotlieb SLD. As condições de saúde no Brasil: restrospecto de 1979 a 1995. Rio de Janeiro: Fiocruz; 2000.

3. Carvalho DMT. Sistema de Informações Hospitalares do SUS - SIH/SUS In: Brasil. Ministério da Saúde. A experiência brasileira em sistemas de informação em saúde. Brasília: Editora do Ministério da Saúde; 2009. p. 49-66.

4. Organização Mundial de Saúde. The World Health Report 2001 - Mental health: new understanding, new hope. Geneva; 2001

5. Organização Mundial de Saúde. The global burden of disease: 2004 update. Geneva; 2008.

6. Organização Mundial de Saúde. Atlas: mental health resources in the world 2001.Geneva; 2001.

7. Kilsztajn S, Lopes ES, Lima LZ, Rocha PAF, Carmo MSN. Leitos hospitalares e reforma psiquiátrica no Brasil. Cad Saúde Pública. 2008;24(10):2354-62.

8. Candiago RH, Abreu PB. Use of Datasus to evaluate psychiatric inpatient care patterns in Southern Brazil. Rev Saude Publica. 2007;41(5):821-9.

9. Silva EC, Costa Junior ML. Transtornos mentais e comportamentais no sistema de informações hospitalares do SUS: perspectivas para a enfermagem. Rev Esc Enferm USP. 2006;40(2):196-202.

10. Coutinho ESF, Klein CH, Morgado AF, Iguchi T, Souza ER. Confiabilidade do diagnóstico psiquiátrico em hospitais do Rio de Janeiro. J Bras Psiquiatr. 1998;37:197-200.

11. American Psychiatric Association. Diagnostic and statistical manual of mental disorders. Washington DC; 1987.

12. Almeida MF, Alencar GP. Informações em saúde: necessidade de introdução de mecanismos de gerenciamento dos sistemas. IESUS. 2000;9(4):241-9.

13. Risi Junior JB. Informações em saúde no Brasil: a contribuição da Ripsa. Ciênc Saúde Coletiva. 2006;11(4):1049-53.

14. Amarante P, organizador. Saúde mental e atenção psicossocial. Rio de Janeiro: FIOCRUZ; 2007.

15. Brasil. Projeto de Lei $\mathrm{n}^{\circ} 3.657$ de 12 de setembro de 1989. Dispõe sobre a extinção progressiva dos manicômios e sua substituição por outros recursos assistenciais e regulamenta a internação psiquiátrica compulsória. Brasília: Câmara dos Deputados; 1991.

16. Brasil. Lei $n^{\circ} 10216$ de 6 de abril de 2001. Dispõe sobre a proteção e os direitos das pessoas portadoras de transtornos mentais e redireciona o modelo assistencial em saúde mental. Diário Oficial da União 2001; 6 abr.

17. Brasil. Ministério da Saúde. Secretaria de Atenção à Saúde. Legislação em saúde mental: 1990-2004. Brasília: Ministério da Saúde; 2004.

18. Brasil. Tribunal de Contas da União. Avaliação das ações de atenção à saúde mental: Programa Atenção à Saúde de Populações Estratégicas e em Situações Especiais de Agravos. Brasília; 2005.

19. Zambenedetti G, Perrone CM. O processo de construção de uma rede de atenção em saúde mental: desafios e potencialidades no processo de reforma psiquiátrica. Physis. 2008;18(2):277-93.
20. Gastal FL, Leite SO, Fernandes FN, Borba AT, Kitamura CM, Binz MAR, Amaral MT. Reforma psiquiátrica no Rio Grande do Sul: uma análise histórica, econômica e do impacto da legislação de 1992. ver Psiquiatr Rio Gd Sul. 2007;29(1):119-29.

21. Brasil. Ministério da Saúde. Coordenação Geral de Saúde Mental. Informativo de Saúde Mental, ano III, n. 17. Brasília; 2004.

22. Botega NJ. Psychiatric units in Brazilian general hospitals: a growing philantropic field. Int J Soc Psychiatry. 2002;48(2):97-102.

23. Larrobla C, Botega NJ. Restructuring mental health: a South American survey. Soc Psychiatry Psychiatr Epidemiol. 2001;36(5):256-9.

24. Botega NJ. Psiquiatria no hospital geral: históricos e tendências. In: Botega NJ, editor. Prática psiquiátrica em hospital geral: interconsulta e emergência. 2. ed. Porto Alegre: Artes Médicas; 2006.

25. Brasil. Ministério da Saúde. Secretaria de Atenção à Saúde. Departamento de Ações Programáticas Estratégicas. Coordenação Geral de Saúde Mental, Álcool e Outras Drogas. Saúde Mental em Dados 9. Brasília; 2011.

26. Freire FHMA, Ugá MAD, Amarante P. Os centros de atenção psicossocial e o impacto do sistema de financiamento no modelo assistencial. In: Amarante P, organizador. Archivos de saúde mental e atenção psicossocial. Rio de Janeiro: NAU; 2005. p. 113-42.

27. Brasil. Conselho Nacional de Saúde. Comissão Organizadora da IV Conferência Nacional de Saúde Mental - Intersetorial. Relatório final da IV Conferência Nacional de Saúde Mental - Intersetorial, 27 de junho a 1 de julho de 2010. Brasília; 2010.

28. American Psychiatric Association. Manual diagnóstico e estatístico de transtornos mentais. 4. ed. Porto Alegre: Artmed; 2002.

29. Mari JJ, Leitão RJ. A epidemiologia da esquizofrenia. Rev Bras Psiquiatr. 2000;22(1):15-7.

30. Gomes MPC, Couto MCV, Pepe VLE, Almeida LM, Delgado PGG, Coutinho ESF. Censo dos pacientes internados em uma instituição asilar no Estado do Rio de Janeiro: dados preliminares. Cad Saúde Pública. 2002;18(6):1803-7.

31. Silva JPL, Coutinho ESF, Amarante PD. Perfil demográfico e sócioeconômico da população de internos dos hospitais psiquiátricos da cidade do Rio de Janeiro. Cad Saúde Pública.1999;15(3):505-11.

32. Andrade L, Walters EE, Gentil V, Laurenti R. Prevalence of ICD-10 mental disorders in a catchment area in the city of São Paulo, Brazil. Soc Psychiatry Psychiatr Epidemiol. 2002;37(7):316-25.

33. Brasil. Ministério da Saúde. A política do Ministério da Saúde de atenção integral a usuários de álcool e outras drogas. 2a ed. Brasília; 2004.

34. Gallassi AD, Elias PEM, Andrade AG. Caracterização do gasto SUS com internações de dependentes de substâncias psicoativas no período de 2000 a 2002 no município de Campinas - SP. Rev Psiquiatr Clín. 2008;35(1):2-7.

35. World Health Organization. Women and health: today's evidence tomorrow's agenda. Geneva; 2009.

36. Andrade LHSG, Viana MC, Silveira CM. Epidemiologia dos transtornos psiquiátricos na mulher. Rev pPsiquiatr Clín. 2006;33(2):43-54.

37. Kessler RC. Epidemiology of women and depression. J Affect Disord. 2003;74(1):5-13 
38. Bennett HA, Einarson A, Taddio A, Koren G, Einarson TR. Prevalence of depression during pregnancy: systematic review. Obstet Gynecol. 2004;103(4):698-709.

39. Korkeila JA, Lehtinen V, Tuori T, Helenius H. Patterns of psychiatric hospital service use in Finland: a national register study of hospital discharges in the early 1990s. Soc Psychiatry Psychiatr Epidemiol.1998;33(5):218-23.

40. Kessler RC, McGonagle KA, Zhao S, Nelson CB, Hughes M, Eshleman S, Wittchen HU, Kendler KS. Lifetime and 12-month prevalence of DSM-III-R psychiatric disorders in the United States: results from the National Comorbidity Survey. Arch Gen Psychiatry. 1994;51(1):8-19.

41. Stoll AL, Tohen M, Baldessarini RJ, Goodwin DC, Stein S, Katz S, Geenens D, Swinson RP, Goethe JW, McGlashan T. Shifts in diagnostic frequencies of schizophrenia and major affective disorders at six North American psychiatric hospitals, 1972-1988. Am J Pschiatry. 1993;150(11):1668-73.

42. Schneid-Kofman N, Sheiner E, Levy A. Psychiatric illness and adverse pregnancy outcome. Int J Gynaecol Obstet. 2008;101(1):53-6.
43. Webb RT, Pickles AR, King-Hele SA, Appleby L, Mortensen PB, Abel KM. Parental mental illness and fatal birth defects in a national birth cohort. Psychol Med. 2008,38(10):1495-503.

44. Weiser M, Kanyas K, Malaspina D, Harvey PD, Glick I, Goetz D, Karni O, Yakir A, Turetsky N, Fenniq S, Nahon D, Lerer B, Davidson M. Sensitivity of ICD-10 diagnosis of psychotic disorders in the Israeli National Hospitalization Registry compared with RDC diagnoses based on SADS-L. Compr Psychiatry. 2005,46(1):38-42.

45. Lyons JS, O'Mahoney MT, Miller SI, Neme J, Kabat J, Miller F. Predicting readmission to the psychiatric hospital in a managed care environment: implications for quality indicators. Am J Psychiatry. 1997;154(3):337-40.

46. Mello-Santos C, Bertolote JM, Wang YP. Epidemiologia do suicídio no Brasil (1980-2000): caracterização das taxas de suicídio por idade e gênero. Rev Bras Psiquiatr. 2005;27(2):131-4.

47. Instituto Brasileiro de Geografia e Estatística. Pesquisa nacional por amostragem de domicílios 2003. Rio de Janeiro; 2005.

Recebido em: 24/05/2012 Aprovado em: 25/08/2012 